\title{
EVALUACIÓN DE IT'S MY TURN, UNA HERRAMIENTA DE AUTOAPRENDIZAJE DE INGLÉS COMO LENGUA EXTRANJERA PARA CONTEXTOS RURALES DE CHILE ${ }^{1}$
}

\author{
Andrea Lizasoain², Amalia Ortiz de Zárate ${ }^{3}$, \\ Mauricio Véliz ${ }^{4}$, Andrea Luci ${ }^{5}$, Julio Rojas-Mora ${ }^{6}$
}

RESUMEN

En este trabajo se informan los resultados de una investigación cuyo objetivo fue comprobar la eficacia del programa estatal It's my Turn, implementado por el Ministerio de Educación de Chile, para el autoaprendizaje del inglés en contextos rurales de todo el país. Se midió el aprendizaje de 120 estudiantes de 10 escuelas rurales de la Región Metropolitana (RM) con pruebas de entrada y salida. Asimismo, se realizaron observaciones para examinar la utilización del recurso, y un cuestionario a los docentes para conocer su percepción del programa. Una prueba $t$ arrojó una diferencia significativa entre las pruebas de entrada y salida ( $p<0,0001)$, lo que respalda la efectividad del programa. Las observaciones pusieron en evidencia métodos heterogéneos de aplicación y los cuestionarios mostraron que los profesores tienen diferentes perspectivas acerca de la eficacia de It's my Turn. La combinación de resultados cuantitativos y cualitativos apunta a la efectividad del programa, pero también a la necesidad de realizar cambios en su implementación.

Palabras clave: autoaprendizaje, inglés como lengua extranjera, aprendizaje de una lengua extranjera, educación rural.

\section{EVALUATIONOF IT'S MYTURN, A SELF-LEARNING INTERACTIVE TOOL TO STUDY ENGLISH IN CHILEAN RURAL CONTEXTS}

\section{ABSTRACT}

This paper presents the results of a study that tested the effectiveness of a self-learning program implemented by the Chilean Ministry of Education, It's my Turn, to promote English learning in rural contexts, throughout Chile. Student learning was measured in 10 rural schools (N students $=120$ ) in the Metropolitan Region (Santiago Capital). Pre- and post-tests were used to measure learning; observations were carried out to examine the actual use of the learning resource in the classroom; and questionnaires were applied to find out the teachers' perceptions of the program. A t-test yielded a significant difference between the pre- and post-tests $(p<0,0001)$. Classroom observations evidenced that teachers used the program in various ways, and the questionnaires showed that teachers have different views on the program's effectiveness. The combination of quantitative and qualitative results leads to the conclusion that the program does favor learning, but also suggests the need for changes in its implementation.

Keywords: self-learning, English as a foreign language, foreign language learning, rural education.

1 Proyecto apoyado financieramente por CNED/ Convocatoria 2013.

2 Universidad Austral de Chile, Instituto de Lingüística y Literatura, Valdivia, Chile. Contacto: andrea.lizasoain@uach.cl

3 Universidad Austral de Chile, Instituto de Lingüística y Literatura, Valdivia, Chile. Contacto: aortizdezarate@uach.cl

4 Universidad Católica Silva Henríquez, Escuela de Educación en Inglés, Santiago, Chile. Contacto: mveliz@usch.cl

5 Universidad San Sebastián, Facultad de Educación, Santiago, Chile. Contacto: andrea.luci@uss.cl

6 Universidad Católica de Temuco, Escuela de Ingeniería Informática, Temuco, Chile. Contacto: julio.rojas@uct.cl 


\section{Introducción}

El inglés se considera la lengua franca por excelencia, pero en Chile muy pocos lo hablan, lo cual priva a sus ciudadanos de comunicarse con otras culturas. Esta realidad es ratificada por las aplicaciones de pruebas estandarizadas desde hace casi una década y por los índices anuales de competencia en inglés del English First (conocidos como EF EPI), que clasifican a Chile en el nivel "muy bajo" de la categorización. En virtud de lo anterior, desde 2004 el Programa Inglés Abre Puertas (PIAP) del Ministerio de Educación de Chile (Mineduc) busca mejorar la calidad de la enseñanza del inglés mediante distintas iniciativas que beneficien a docentes y estudiantes.

Hasta el año 2010, no obstante, las zonas rurales habían quedado ajenas a estos proyectos. Para remediar esta situación, el PIAP propuso la creación de un programa de autoaprendizaje de inglés como lengua extranjera (ILE) para el contexto rural, en el que escaseaban docentes de la especialidad. Atendiendo a este vacío, un grupo de académicos chilenos diseñó It's my Turn (ImT), un programa que permitiría a los estudiantes rurales aprender la lengua inglesa de forma autónoma, entretenida y eficiente en términos de tiempo, espacio y recursos. La idea original era que los estudiantes aprendieran inglés sin quitarles protagonismo a los profesores; no obstante, en la práctica, muchos docentes se convirtieron en un estudiante más, debido a que no tienen nociones del idioma (Mineduc, 2016b).

It's my Turn es un recurso audiovisual diseñado para quinto y sexto básico, que consiste en un set de cinco DVD con lecciones pregrabadas, un libro para el estudiante, un libro para el profesor, un $\mathrm{CD}-\mathrm{ROM}$ con canciones y rimas en inglés, un CD-ROM con instrucciones de uso para el profesor, un diccionario, un cuaderno de evaluación con sugerencias para medir el aprendizaje de los contenidos y un cuaderno de planificaciones de todas las unidades.

Las lecciones están diseñadas en torno a dos contextos diferentes pero interrelacionados: una escuela rural de Chile, en la que Míster Campos enseña inglés a sus estudiantes; y un árbol en el patio de la escuela donde viven cuatro animalitos autóctonos: una chinchilla, un pudú, una loica y un armadillo. Estas lecciones avanzan contando la 

INGLÉS COMO LENGUA EXTRANJERA PARA CONTEXTOS RURALES DE CHILE - A. Lizasoain, A. Ortiz de Zárate, M. Véliz, A. Luci y J. Rojas-Mora

historia de un estudiante estadounidense que intenta comunicarse con sus compañeros chilenos. En el set de quinto básico se enfatiza la adquisición de léxico, mientras que en las lecciones de sexto básico se acentúa también la enseñanza de las estructuras lexicogramaticales básicas de la lengua inglesa.

Hasta 2012, como es común en relación con la implementación de programas educacionales nuevos en Chile, no se había comprobado la utilidad del programa. En consecuencia, en el marco de la práctica profesional y seminario de tesis de Pedagogía en Comunicación en Lengua Inglesa (Becchi, 2012), se diseñó un estudio de caso de pequeña escala para evaluar la efectividad del recurso en tres escuelas rurales de la Región de los Ríos. Sin embargo, las escuelas participantes no utilizaban el programa con regularidad -pese a haber declarado que sí lo hacían-, por lo que el estudio de caso se convirtió en una investigación-acción a través de la cual se implementó el programa para medir los avances de los estudiantes durante un mes. Se encontró que este era efectivo, es decir, los estudiantes aprendían inglés a través de ImT (Lizasoain \& Becchi, 2014).

El presente estudio surgió de la necesidad de validar los resultados de 2012 a mayor escala, teniendo en cuenta el alto porcentaje de escuelas rurales en nuestro país. Según estadísticas del Mineduc, el número asciende a cerca de 4.345, lo que representa alrededor de un 50\% del total de establecimientos educacionales (Mineduc, 2014) ${ }^{7}$ en Chile. Para hacerlo, se midieron los conocimientos adquiridos durante un semestre de exposición a ImT de estudiantes de escuelas rurales de la RM. Asimismo, se exploró la perspectiva de los profesores rurales acerca del programa en cuanto a su utilidad, facilidad de uso, efectividad y disponibilidad de materiales.

A continuación, se contextualiza el problema en Chile y se exponen las bases teóricas de ImT. Luego se describen la metodología y los resultados. Finalmente, se discuten los datos a la luz del problema que representa la enseñanza-aprendizaje del inglés en Chile en general y en los contextos rurales en particular.

7 Esta base de datos ya no es de libre acceso. 


\section{Estado de la cuestión: el inglés en las aulas chilenas}

Desde 2004, el Ministerio de Educación de Chile ha aplicado regularmente pruebas estandarizadas para evaluar la comprensión escrita y oral en inglés que poseen estudiantes de educación media de todas las regiones y tipos de establecimientos educacionales, excepto aquellos de zonas rurales. En 2010, estas pruebas se convirtieron en la medición Simce Inglés (Sistema de Medición de la Calidad de la Educación), que se aplica cada dos años en tercero medio. Los resultados han arrojado sistemáticamente que los alumnos no alcanzan el nivel de competencia comunicativa que el Mineduc exige en la cobertura curricular para tercero medio, a saber A2 o B1, según el Marco Común Europeo de Referencia para las Lenguas (MECR). De contar con estos niveles, los estudiantes podrían entender las ideas principales de textos simples relacionados con el trabajo, la escuela o entretención, o de comunicarse en situaciones de viaje. En otras palabras, al terminar la enseñanza media, los futuros profesionales y técnicos podrían desenvolverse con éxito en situaciones de comunicación básicas.

La evaluación de 2004 incluyó un cuestionario para identificar las prácticas pedagógicas de los profesores. Este arrojó que el rol del docente es esencial para el aprendizaje del inglés. De hecho, aquellos estudiantes que tenían la oportunidad de participar en múltiples actividades de aprendizaje y evaluación orales y escritas obtuvieron mejores niveles de competencia comunicativa. Al mismo tiempo, los estudiantes cuyos profesores efectivamente interactuaban en inglés en el aula aprendieron la lengua extranjera y, por lo tanto, tuvieron éxito en las pruebas estandarizadas. Por el contrario, los estudiantes que no estaban expuestos a otra lengua -la gran mayoría- obtuvieron resultados bajos (Lizasoain \& Ortiz de Zárate, 2009).

Lo anterior se condice con Sun, Calderón, Valerio y Torres (2011) en cuanto a que en Chile "la mayor parte de los docentes [de inglés] establecen interacciones con sus alumnos que no favorecen el desarrollo del pensamiento o no constituyen un aporte significativo para enriquecer su aprendizaje" (p. 121). Además, "los profesores 
102 EVALUACIÓN DE IT'S MY TURN, UNA HERRAMIENTA DE AUTOAPRENDIZAJE DE INGLÉS COMO LENGUA EXTRANJERA PARA CONTEXTOS RURALES DE CHILE - A. Lizasoain, A. Ortiz de Zárate, M. Véliz, A. Luci y J. Rojas-Mora

[de inglés] tienden a basar su enseñanza únicamente en los textos de estudio, dejando muy poco tiempo para experiencias creativas" (Lizasoain \& Ortiz de Zárate, 2009, p. 2), así como para el uso de material auténtico (Lizasoain, Ortiz de Zárate, Walper \& Yilorm, 2011) y tecnologías de la información y la comunicación (Arancibia, 2002; Cárcamo, Cladellas y Castelló, 2011; Flores, 2012; Pérez, 2000). Lamentablemente, en Chile las clases solo "estimulan la actividad corporal de estar sentados en filas [y] suponen las actividades de leer, escribir, escuchar y responder a las preguntas del profesor" (Contreras, 2002, citado en Lizasoain \& Ortiz de Zárate, 2009, p. 2). Esto se traduce en la participación pasiva del estudiante en el proceso de aprendizaje y la falta de adquisición real de conocimientos.

Sumado a esto, se ha evidenciado que la competencia comunicativa de los profesores del área está por debajo del nivel B2 del MECR, exigido para los docentes hasta 2013. El nivel B2 clasifica al hablante de lengua extranjera como aquel que comprende y produce textos complejos en distintos contextos de la realidad educacional y social. Además, un hablante B2 puede comunicarse con fluidez y claridad con un interlocutor nativo, y expresar rasgos de su personalidad a través de la lengua extranjera. No obstante, al no contar con esta competencia, los docentes difícilmente pueden fomentar el intercambio de ideas de forma natural en el aula.

En relación con esta realidad y en un esfuerzo por mejorar la competencia comunicativa a través del perfeccionamiento docente, en 2014 el Ministerio de Educación -en conjunto con las universidades chilenas que imparten la carrera- decidió proponer nuevos estándares orientadores para la carrera de Pedagogía en Inglés. Esto con el objeto de mejorar la competencia comunicativa de los futuros profesores y de los docentes en ejercicio a un nivel C1, superior al B2. En el C1, el hablante puede comprender y producir textos extensos de diversos temas no necesariamente relacionados con su especialidad, así como expresarse mediante un discurso coherente y cohesivo según un contexto dado. En otras palabras, a diferencia del nivel B2, el hablante no solo se desenvuelve respecto de temas que le son familiares, sino que puede improvisar en situaciones de comunicación tanto especializadas como cotidianas. De esta manera, se puede fomentar 
el intercambio real, que no solo exige hablar acerca de temas propios de una disciplina, sino que también discutir, negociar, bromear, etc. No obstante, cabe preguntarse ¿cómo se va a formar a un profesor de inglés con nivel $\mathrm{Cl}$, si hasta ahora las universidades no han sido capaces de formar profesores con nivel B2?

Los Estándares Orientadores dan cuenta de la competencia comunicativa y disciplinar con la que deben contar los profesores de la especialidad, y que orientan la formación docente. Entre estos estándares se encuentran el conocimiento cabal de la lengua inglesa, capacidad de desarrollar las cuatro habilidades básicas de una lengua (escuchar, leer, hablar y escribir), competencia comunicativa avanzada (nivel $\mathrm{Cl}$ ) y conocimiento de teorías de aprendizaje, entre otros (Mineduc, 2016c). A continuación, se hace referencia a algunas teorías de aprendizaje y metodologías de enseñanza que los profesores de inglés debieran conocer, y que competen a este trabajo por conformar las bases teóricas de It's my Turn.

\section{Marco teórico: enseñanza-aprendizaje del inglés como lengua extranjera}

\subsection{Aprendizaje y adquisición}

El concepto de aprendizaje de una lengua extranjera (LE) tiende a referirse al dominio de una lengua que no es la materna (L1) en un país donde la LE no es oficial. La adquisición, en cambio, se relaciona con una segunda lengua (L2), la que se adquiere -y no se aprende-en un país donde esta sí es oficial (Krashen, 1981). Por ejemplo, si un chileno estudia francés en Francia, puede adquirir la L2; en cambio, si estudia francés en Chile, puede aprender la LE. Si bien es cierto una LE se aprende generalmente en entornos formales como el aula -en comparación con contextos más informales como la calle-, según algunos expertos, el proceso de adquisición de la Ll se puede emular hasta cierto punto, si se cumple con ciertos requisitos.

\subsubsection{Enfoque Natural}

Desde el Enfoque Natural (Krashen \& Terrel, 1977), se entiende que la adquisición del idioma extranjero en el aula puede fomentarse 
104 EVALUACIÓN DE IT'S MY TURN, UNA HERRAMIENTA DE AUTOAPRENDIZAJE DE INGLÉS COMO LENGUA EXTRANJERA PARA CONTEXTOS RURALES DE CHILE - A. Lizasoain, A. Ortiz de Zárate, M. Véliz, A. Luci y J. Rojas-Mora

siguiendo la misma lógica que cuando se aprende la lengua materna. De esta forma, en el aula se enfatiza la fluidez de la comunicación por sobre la corrección, se enseña vocabulario y estructuras sintácticas de forma implícita y se expone a los estudiantes a un input auténtico y comprensible (Krashen, 1985). Se espera que puedan integrar este input de forma inconsciente, de la misma forma en que lo hacen los niños cuando aprenden a hablar su Ll. Así, en lugar de aprender acerca de la lengua, como es común en los contextos formales, los estudiantes aprenden a usarla; es decir, la adquieren.

El aprendizaje y la adquisición, no obstante, están intrínsecamente relacionados. Pese a que en algunos contextos existe la tendencia a desacreditar el aprendizaje en virtud de la adquisición, aquí lo entendemos como paralelo a esta última. En esta línea, Krashen (1981) reconoce que los aprendientes tienen dos sistemas independientes, pero interrelacionados que son igualmente potentes para aprender una LE/L2, a saber, el aprendizaje consciente y la adquisición inconsciente. El aprendizaje es una etapa inmediatamente previa y quizás paralela a la adquisición. A modo de ejemplo, aprender a conducir un automóvil implica un esfuerzo consciente por controlar el volante, los cambios, las luces, etc. Una vez dominados estos elementos, el aprendizaje se convierte en adquisición y ya no es necesario concentrarse en cada uno, sino que se mecanizan. En otras palabras, la adquisición es el resultado de la automatización del aprendizaje.

Lantolf (2013) da cuenta de lo anterior al señalar que en el aula no es siempre posible emular la adquisición de la L1. Es más, el autor concibe la educación como "una orquesta de actividades" (p. 27) dirigidas por el profesor para introducir "conocimiento explícito" (p. 28). En el contexto de enseñanza de una lengua extranjera, es la enseñanza la que conduce al aprendizaje, el cual es mediado por el profesor (Lantolf, 2013). Una vez que el estudiante aprende, puede adquirir. De esta forma, si el profesor es responsable del aprendizaje, la adquisición es responsabilidad de los estudiantes, la que debe fomentarse mediante la producción -o creación de conocimiento nuevo- a partir de lo aprendido. 


\subsubsection{Enfoque Comunicativo}

El Ministerio de Educación chileno promueve la enseñanza del inglés como lengua extranjera desde el Enfoque Comunicativo que, basado en el Enfoque Natural, persigue el desarrollo de una lengua mediante el uso significativo del lenguaje en el aula (Richards \& Rogers, 2008). Este uso significativo tiene dos aristas. En primer lugar, se busca que el aprendiente relacione el conocimiento nuevo con sus experiencias en el mundo (aprendizaje). En segundo lugar, se espera que este pueda utilizar el conocimiento nuevo y cumplir sus propósitos comunicativos (adquisición). Esto último es lo que se conoce como competencia comunicativa: la capacidad del hablante de utilizar el lenguaje con fines comunicativos según un contexto dado.

El programa It's my Turn, apunta al desarrollo seminal de la competencia comunicativa. Su objetivo es lograrla basándose mayormente en el aprendizaje/adquisición de léxico en quinto básico, nivel con el que se trabajó. En primera instancia, en el aula se presentan los contenidos de manera explícita, representando elementos de la vida cotidiana tanto en inglés como en español. Estos dicen relación con tres áreas generales del conocimiento de una lengua, a saber: léxico básico (colores, frutas, mascotas, partes del cuerpo); recursos gramaticales (preposiciones, verbos, adjetivos); y funciones comunicativas (saludar, presentarse, expresar gusto). Lo anterior, con el propósito de permitir al aprendiente etiquetar una realidad que ya conoce con términos nuevos. De esta manera, aparecen expresiones como "Mira, esta es una manzana/This is an apple" o "Aprendamos los colores en inglés: white, blue, red, green...", que tienen la intención de promover el aprendizaje (consciente) primero y la adquisición (inconsciente) después.

\subsubsection{Instrucción Basada en Contenidos}

En relación con el uso significativo de elementos incorporados al repertorio en inglés, otro enfoque que inspira al programa It's my Turn es el de Instrucción Basada en Contenidos (IBC). Esta promueve la adquisición del inglés en torno a temáticas relacionadas con otras asignaturas del plan educativo (Arancibia, 2002; Stryker \& Leaver, 1997), de modo de evitar conocimientos aislados por 

INGLÉS COMO LENGUA EXTRANJERA PARA CONTEXTOS RURALES DE CHILE - A. Lizasoain, A. Ortiz de Zárate, M. Véliz, A. Luci y J. Rojas-Mora

un lado y contextualizar el lenguaje, por otro. Por ejemplo, se han incorporado animales autóctonos de distintas zonas del país, los que se presentan con una manufactura audiovisual que resulte atractiva para los estudiantes de este curso. Asimismo, el guion del programa se desarrolla en torno a la llegada de un chico estadounidense con el cual los estudiantes rurales chilenos intentarán comunicarse e integrar a su realidad. Así, se intenta que estos últimos perciban el aprendizaje del inglés como una necesidad, así como la posibilidad de su utilización inmediata y real. Esta percepción no solo se fomenta a través de la observación de la relación entre el estudiante extranjero y sus compañeros de clase, sino también a través de la interacción del material audiovisual por parte de los estudiantes. Respecto de esto último, antes, durante y al finalizar las lecciones, la clase debe contestar preguntas presentadas por los personajes del programa, o bien, completar información de forma oral y escrita. De esta manera, se privilegia lo educativo antes que lo tecnológico (Chiappe, 2009). En otras palabras, la herramienta se presenta como material educativo, de la misma manera que un libro de texto o una pizarra.

Finalmente, además de apuntar al aprendizaje/adquisición de elementos lexicogramaticales del inglés, mediante la presentación de conocimientos nuevos ligados a una realidad conocida, It's my Turn recurre a otro elemento atractivo, familiar y productivo para los aprendientes: las TIC, a las que nos referimos a continuación.

\subsubsection{Aprendizaje Basado en TIC}

El uso de recursos de Tecnologías de la Información y Comunicación (TIC) en la educación se sustenta en la premisa de que estas permiten el acceso de cualquier niño, rural o urbano, a más información de la que podría haber accedido cualquier científico del siglo pasado (Warschauer, Shetzer \& Meloni, 2004). Fruto de un cambio cultural innegable en el nivel global, el uso de las TIC es hoy imprescindible (Castells, 1997). En esta línea, los estudiantes actuales se consideran "nativos digitales" (Prensky, 2001), screenagers (Jukes \& Dosaj, 2006) o "residentes digitales" (White \& Le Cornu, 2010). Esto significa que exploran, conocen y aprenden con naturalidad a través de escenarios virtuales (Fernández, 2011). En este sentido, el uso de computadores, 
teléfonos inteligentes, televisión, cuentas de correo electrónico, redes sociales, etc., parece ser ineludible hoy para el logro de aprendizajes significativos y de largo plazo (Chiappe, 2009).

Según Hepp (2013), las TIC fomentan la autonomía en el aprendizaje haciendo que los estudiantes sean más responsables de su propio desarrollo escolar y académico. Dicho de otra forma, liberan al profesor de toda la responsabilidad del aprendizaje (García, Ferrerira y Morales, 2012). Un punto intermedio entre estas visiones es el de Chiappe (2009), quien plantea que las TIC son un recurso de mediación del aprendizaje. Es decir, como un intermediario entre lo que se ha de aprender y el aprendiente. Este intermediario se presenta "como un cierto material educativo digital" (Chiappe, 2009, p. 262), cuyo objetivo es hacer que alguien aprenda algo.

Dicho aprendizaje se logra, según Álvarez y Álvarez (2012), gracias a la naturaleza mixta de los mensajes verbales y visuales de las TIC, la cual favorece el almacenamiento de información y, en consecuencia, facilita el mismo. Esto porque "hay más posibilidades de que se recuerde la información si se presenta en dos formatos" diferentes (Álvarez y Álvarez, 2012, p. 222). No obstante lo anterior, las TIC no se reducen a un artefacto de almacenamiento de la información, sino que más bien constituyen una extensión de la mente humana, fruto de la relación entre el hombre y la tecnología (Cárcamo, Cladellas \& Estaún, 2007). Esta extensión ha generado un cambio social que convierte a la sociedad en una "sociedad red" (Castells, 1997), lo que trasciende a sus esferas educacionales, laborales, comunicacionales y políticas, entre otras. En consecuencia, las TIC no constituyen recursos de fomento, mediación y almacenamiento de información y conocimientos únicamente, sino que representan una forma de ser y actuar del hombre de hoy (Castells, 1997). En este sentido, su inclusión en la escuela es un fenómeno natural, el cual se traduce en una manera de acercarse, apropiarse y producir conocimiento, así como en una forma de desarrollar habilidades sociales.

A modo de resumen, It's my Turn se basa en un enfoque de enseñanza de lenguas extranjeras que busca fomentar el aprendizaje de 
recursos lexicogramaticales básicos fundamentales para la adquisición, presentando el contenido lingüístico -o input- de manera explícita e implícita, con la intención de que los estudiantes lo aprendan de forma consciente y lo apliquen luego de inconscientemente. Además, fomenta el aprendizaje significativo del inglés, porque el contenido lingüístico se presenta inserto en un contexto conocido para los estudiantes rurales a través de un recurso de mediación del aprendizaje que hoy en día resulta natural en el sistema educacional.

\section{Método de investigación}

Esta propuesta es un estudio de caso evaluativo (Cohen, Manion \& Morrison, 2007; Stenhouse, 1983) que buscó medir la efectividad de un programa de autoaprendizaje de inglés en zonas rurales utilizando los resultados de pruebas de entrada y salida, observaciones en aula y cuestionarios a docentes. Se llevó a cabo en los quintos básicos de 10 escuelas rurales de la Región Metropolitana, incluidos estudiantes y profesores.

La hipótesis nula planteó que después del tratamiento con el programa It's my Turn no habría diferencia entre los resultados iniciales y los posteriores. La hipótesis alternativa decía relación con una diferencia estadísticamente significativa $(p<0,05)$ entre estos resultados.

\subsection{Participantes}

Las escuelas se seleccionaron a partir de la base de datos disponible en línea del Ministerio de Educación (data.mineduc.cl) y se invitaron a participar mediante correo electrónico o contacto telefónico. Debido a que de esta manera no se consiguió la cantidad de escuelas deseadas, se solicitó ayuda al Mineduc para hacer de puente con otros establecimientos. Así se logró contar con 10 escuelas rurales públicas de cuatro comunas de la Región Metropolitana: El Monte, Isla de Maipo, María Pinto y San José de Maipo. Para proteger la identidad de las escuelas, se cambiaron sus nombres por otros que mantienen una sutil similitud con los originales. 


\subsubsection{Profesores}

Participaron siete profesores, todos los cuales contaban con certificación en la especialidad, es decir, eran profesores de inglés o habían realizado los cursos de ILE ofrecidos por el Ministerio de Educación. Seis eran de sexo femenino y uno masculino. Además, contaban con un nivel al menos intermedio de inglés (B1), lo que significa que podían comunicarse en esta lengua en cualquier situación escolar dentro del aula (saludar, dar instrucciones, explicar, llamar la atención, etc.). Cabe destacar que en la comuna de Isla de Maipo la misma profesora enseñaba en las tres escuelas participantes; en la comuna de María Pinto, por su parte, un profesor enseñaba en dos de las cinco escuelas participantes.

\subsubsection{Estudiantes}

Participaron 120 estudiantes de quinto básico de entre 10 y 12 años: 67 eran niñas y 53 niños. La Tabla 1 indica la cantidad de estudiantes por escuela. Como se observa, en la mayoría de las aulas la cantidad de estudiantes es pequeña, con un promedio de 12 alumnos por sala, con excepción de una de las escuelas de María Pinto, que tiene un grupo curso similar a los que se encuentran en los establecimientos educacionales de las zonas urbanas. Una de estas escuelas (El Melón) es combinada e integra un quinto y un sexto básico. En este caso, participaron los 13 estudiantes del aula, puesto que el sexto básico no había tenido inglés anteriormente.

Tabla 1: Cantidad de estudiantes por escuela

\begin{tabular}{c|c|c|c|c|c|c|c|c|c|c|c}
\hline Comuna & El Monte & Isla de Maipo & \multicolumn{4}{|c|}{ María Pinto } & \multicolumn{3}{c|}{ San José de Maipo } & \\
\hline Escuela & Jesucristo & San Andrés & Chobo B & Chobo A & $\begin{array}{c}\text { Santa } \\
\text { Ana }\end{array}$ & $\begin{array}{c}\text { Los } \\
\text { Milagros }\end{array}$ & $\begin{array}{c}\text { Las } \\
\text { Huertas }\end{array}$ & $\begin{array}{c}\text { El } \\
\text { Ciruelo }\end{array}$ & $\begin{array}{c}\text { El } \\
\text { Romero }\end{array}$ & $\begin{array}{c}\text { El } \\
\text { Melón }\end{array}$ & Total \\
\hline N. ${ }^{0}$ & 9 & 8 & 9 & 28 & 16 & 10 & 14 & 6 & 7 & 13 & 120 \\
\hline
\end{tabular}

\subsection{Herramientas de investigación}

\subsubsection{Pruebas de entrada y salida}

Las evaluaciones de entrada y salida se aplicaron al comienzo y al término del primer semestre de 2014, respectivamente. La evaluación de entrada recopiló información de los conocimientos previos a las 
unidades a trabajar según el cuaderno de planificación de la batería de $\operatorname{ImT}$. Se midieron los contenidos que debieran ser aprendidos durante el primer semestre, es decir, algunas preposiciones, los números, los colores, los miembros de la familia, diálogos básicos, elementos de la sala de clases y días de la semana.

La evaluación de salida midió el logro de los estudiantes respecto de los mismos contenidos. Las pruebas fueron similares entre sí en términos de contenidos y nivel de dificultad, adaptadas del cuaderno de evaluación del programa. Esto con el fin de que existieran parámetros de comparación que permitieran el análisis de datos (Gass $\&$ Mackey, 2012) y que tuvieran validez de constructo (Brown, 2004). Se eliminaron los datos de aquellos estudiantes que no rindieron ambas pruebas. Con el fin de evitar que los estudiantes recordaran los ítems de la evaluación inicial y los respondieran mecánicamente -lo que invalidaría los datos (Gass \& Mackey, 2012)-, las pruebas fueron diferentes en términos de formulación. Las instrucciones se presentaron en inglés y en español, al igual que en las evaluaciones prediseñadas del cuaderno de evaluación.

El puntaje total final de ambas pruebas fue de 108 puntos. Las respuestas se consideraron parcialmente correctas si reflejaban la pronunciación de las palabras en inglés. Por ejemplo, si one estaba escrito como "wan", se otorgó medio punto. Esto porque el trabajo en aula integra tanto la práctica oral como el trabajo escrito (en los libros de textos, cuadernos de los estudiantes y pizarra), por lo que se asumió que los estudiantes también debían conocer la grafía de las palabras.

\subsubsection{Pautas de observación}

Se realizaron dos o tres observaciones en aula durante el primer semestre de 2014, entre marzo y junio, cada una de entre 45 y 90 minutos $^{8}$. Para ello se utilizó una adaptación de la pauta de observación no participativa Communicative Orientation of Language

8 En los establecimientos públicos y subvencionados, las horas pedagógicas destinadas al sector inglés son tres, por lo que en ocasiones se observaron ventanas de 45 minutos y, en otras de 90 minutos, de acuerdo con la disponibilidad de tiempo de los investigadores y profesores. 
Teaching (COLT, Orientación Comunicativa de la Enseñanza de Idiomas en español) de Flyman-Mattsson (1999). Esta permite describir en detalle algunas características de la comunicación que tienen lugar en un contexto de enseñanza de segundas lenguas, como las actividades, la organización de los participantes, el contenido y los recursos utilizados (Nunan, 1992). Se buscó conocer in situ la metodología de aplicación del recurso digital y el desarrollo de las actividades con los estudiantes.

Esta pauta de observación registra los tipos de actividades que se desarrollan en el aula, como lectura, escritura, trabajo colaborativo, etc. También se señala la organización del grupo clase (toda la clase, grupos o individual), tipo de contenido trabajado en cada actividad (gramática, vocabulario, otro) y utilización de ImT, entre otros aspectos.

\subsubsection{Cuestionario}

Al término del período de observación y aplicación de la prueba de salida (fines de junio y comienzos de julio de 2014), se aplicó un cuestionario en línea con soporte en Survey Monkey, con el fin de conocer la opinión de los docentes rurales participantes acerca del impacto que tiene el programa de inglés rural en el proceso de aprendizaje de los estudiantes, su percepción de las capacitaciones entregadas por el Mineduc para la implementación del programa, así como las posibles mejoras a incorporar.

\subsection{Análisis de datos}

Las pruebas de entrada y salida fueron corregidas por dos investigadoras con la misma pauta de corrección y la misma escala de notas, calculadas en el generador de escala de notas de Pumarino (2016). La exigencia para la nota 4,0 fue del $60 \%$, al igual que el porcentaje de exigencia que en las escuelas. Se utilizó una prueba $t$ de muestras dependientes de dos colas para comparar los resultados de las pruebas de entrada y salida de cada una de las escuelas y comprobar si la diferencia entre los conocimientos iniciales y los logrados después de un semestre era significativa. Se aplicó una prueba de dos colas, asumiendo que no habría una diferencia significativa (Hatch \& Farhady, 1982). 
El análisis de los datos cualitativos de las observaciones en aula fue de tipo genérico (Creswell, 2009) y se describieron bajo los criterios "Uso de It's my Turn" y "Utilización de L1 y L2 en el aula". Después de cada observación, se sistematizó esta información en forma de narraciones descriptivas (análisis genérico de Creswell). Además, se hicieron comparaciones y reflexiones acerca de las clases dadas por un mismo profesor en busca de patrones. De esta manera, siguiendo las sugerencias de Creswell (2009) para el análisis de datos cualitativos, se avanzó al mismo tiempo en la recolección de datos, las interpretaciones de estos y la redacción del presente informe. Los datos se convirtieron en descripciones de cómo se usa $\operatorname{ImT}$ y el grado de utilización de la L1 y L2 en la sala de clases. Debido al bajo porcentaje de respuestas a los cuestionarios, estos se analizaron en términos cualitativos en torno al impacto de $\operatorname{ImT}$ en el aprendizaje y la evaluación del papel del Mineduc con respecto al programa por parte de los docentes.

\section{Resultados}

\subsection{Pruebas de entrada y salida}

La prueba de entrada se aplicó durante las últimas dos semanas de marzo de 2014. Los resultados de las 10 escuelas participantes fueron, en general, bajos, con una media de 2,78 ( $D E=1,15)$. Ningún estudiante logró la nota máxima, pero hubo tres casos con nota mayor a 6,0. Siete de las diez escuelas obtuvieron una media inferior a 3,0. Estos resultados estuvieron dentro de lo esperado, dado que los estudiantes de establecimientos públicos y subvencionados generalmente no tienen inglés antes de quinto básico. Se encontró que muchos de ellos tenían nociones básicas previas de los colores, los números, los miembros de la familia y los elementos de la sala de clases en inglés, pero desconocían nociones más complejas, como las preposiciones, y no producían en inglés en el modo escrito. Además, se observó que los estudiantes tienden a escribir las expresiones como se pronuncian, pese a que el input tiende a ser escrito.

La prueba de salida se aplicó durante las últimas dos semanas de junio y la primera semana de julio de 2014. Al igual que los resultados 
de la prueba de entrada, los de salida fueron, en general, bajos, con una media un poco mayor de 3,42 ( $D E=1,26)$. Ningún estudiante logró la nota máxima, pero nuevamente hubo tres casos con nota mayor a 6,0. Solo uno de estos casos coincide con los de la prueba de entrada. Esta vez, siete de las 10 escuelas obtuvieron una media superior a 3,0 superando las de la prueba de entrada. Se encontró que la mayoría de los estudiantes conoce en inglés los colores, los números, los miembros de la familia, los días de la semana y los elementos de la sala de clases. No obstante, al igual que en la prueba de entrada, se observa que desconocen nociones más complejas y que no producen en inglés en el modo escrito. De hecho, se eliminó el ítem de producción escrita de respuestas cerradas -que no se había incluido en la prueba de entrada-, en la que se les hacían preguntas básicas como "How are you?" y "What is your name?", puesto que la gran mayoría de los estudiantes las dejó en blanco. Además, se observó que estos tienden a escribir las expresiones como se pronuncian en la mayoría de los casos, lo que se evidencia especialmente en la escritura de los números y los elementos de la sala de clases. Por ejemplo, pueden escribir "wan" (one), "shu" (two), "tri" (three), "for" (four), "fai" (five), etc. Al mismo tiempo, escriben "clasrum" (classroom), "bakpak" (back pack) y "teibel" (table).

No obstante lo anterior, todas las escuelas subieron sus resultados, si bien la mayoría no alcanzó la nota suficiente $(4,0)$. La Tabla 2 identifica los resultados de ambas pruebas. Como se observa, la diferencia tuvo significancia estadística en cinco escuelas (en gris). Pese a que los resultados siguen siendo bajos, se observa un avance positivo, sobre todo en el caso de las escuelas Chobo A y Los Huertos, que fueron las que más mejoraron en relación con las demás escuelas ( 1,14 y 1,2 puntos, respectivamente). Asimismo, la escuela San Andrés logró pasar al nivel suficiente $(4,0)$ de rendimiento en la escala de notas de Chile. Cabe destacar aquí que, pese a ser la única escuela de modalidad combinada (quinto y sexto juntos en una sala) de la muestra, la escuela El Melón no se comporta de una manera diferente a las demás, es decir, no obtiene resultados distintos, quizás porque ninguno de los niveles había tenido inglés antes. 
114 EVALUACIÓN DE IT'S MY TURN, UNA HERRAMIENTA DE AUTOAPRENDIZAJE DE INGLÉS COMO LENGUA EXTRANJERA PARA CONTEXTOS RURALES DE CHILE - A. Lizasoain, A. Ortiz de Zárate, M. Véliz, A. Luci y J. Rojas-Mora

Tabla 2: Diferencia de resultados prueba de entrada y salida

\begin{tabular}{|c|c|c|c|c|c|c|c|c|c|c|c|}
\hline Comuna & El Monte & $\begin{array}{l}\text { Isla de } \\
\text { Maipo }\end{array}$ & \multicolumn{5}{|c|}{ María Pinto } & \multicolumn{3}{|c|}{ San José de Maipo } & \\
\hline Escuela & Jesucristo & $\begin{array}{c}\text { San } \\
\text { Andrés }\end{array}$ & $\begin{array}{c}\text { Chobo } \\
\text { B }\end{array}$ & $\begin{array}{c}\text { Chobo } \\
\text { A }\end{array}$ & $\begin{array}{c}\text { Santa } \\
\text { Ana }\end{array}$ & $\begin{array}{c}\text { Los } \\
\text { Milagros }\end{array}$ & $\begin{array}{l}\text { Los } \\
\text { Huertos }\end{array}$ & $\begin{array}{c}\text { El } \\
\text { Ciruelo }\end{array}$ & $\begin{array}{c}\text { El } \\
\text { Romero }\end{array}$ & $\begin{array}{c}\text { El } \\
\text { Melón }\end{array}$ & $x$ \\
\hline Media E & 2,62 & 3,4 & 2,96 & 2,63 & 3,34 & 4,07 & 1,91 & 2,4 & 2,2 & 2,46 & 2,78 \\
\hline Media S & 3,22 & 4,33 & 3,31 & 3,77 & 3,56 & 4,01 & 3,1 & 2,98 & 2,96 & 2,57 & 3,43 \\
\hline P & 0,08 & 0,0003 & 0,23 & 0,0001 & 0,11 & 0,85 & 0,0001 & 0,02 & 0,02 & 0,66 & \\
\hline
\end{tabular}

Para determinar si la diferencia entre los resultados de entrada y salida generales de las 10 escuelas era significativa, se aplicó una prueba $t$, que arrojó una diferencia relevante $(t(119)=-8,15 ; p<$ 0,0001 ). En consecuencia, pese a que los resultados fueron bajos en ambas pruebas, se puede afirmar que el recurso ImT es efectivo para el aprendizaje del inglés. Sin embargo, cabe preguntarse por qué son tan bajos los resultados.

El análisis cualitativo puede dar luces de lo anterior. Durante las observaciones en aula, se encontró que no todos los profesores utilizan el recurso del Mineduc de la misma manera, lo que podría explicar los bajos resultados de las pruebas de salida. A continuación, se detalla el análisis cualitativo proveniente de las observaciones, lo que posteriormente se relaciona con el menor o mayor grado de avance de las escuelas.

\subsection{Observaciones}

Los datos provenientes de las observaciones se analizaron en torno a dos criterios: uso del recurso It's my Turn y utilización de los idiomas inglés y español en el aula. En relación con el uso del recurso TIC, se observaron tres tendencias. Por una parte, existen profesores que basan sus clases únicamente en ImT. Por otra, algunos docentes lo combinan con sus propios recursos y finalmente, hay otros que, pese a declarar que usan el programa, no lo utilizan en absoluto. En la sección de discusión, se examina si estas tendencias tienen algún impacto en el rendimiento de los estudiantes.

Respecto de la utilización del español (L1) y el inglés (LE) en el aula, se encontraron dos tendencias. En primer lugar, existe un 
uso parcial generalizado de la lengua inglesa, lo que significa que los profesores utilizan la Ll para ciertos tipos de interacción, como explicaciones complejas, traducción, descripciones, regaños, etc., mientras que utilizan la LE para saludar, dar instrucciones, hacer preguntas básicas, elogiar y retroalimentar. En este tipo de uso se incluye la traducción inmediata al español de todas las expresiones de los profesores. Por otra parte, se observó un uso sistemático de la LE para impartir la mayor parte de la clase, con la utilización de la Ll solo para casos extremos de incomprensión por parte de los estudiantes o para intercambios relativos a contenidos en general ajenos a la asignatura. Esta constituye la tendencia menos común. A continuación, se presentan tres ejemplos de cada tendencia:

a) Utilización parcial de Ll y LE

- Profesor 1: Millaray, te dije que no siguieras avanzando en el libro. Ciérralo (uso predominante del español).

- Profesor 1: ¿Qué color es green? (uso combinado de español e inglés).

- Profesor 1: Ok, now we are going to watch the video. Ahora vamos a ver el video (traducción del inglés al español).

b) Utilización exclusiva de LE

- Profesor 4: Please, be quiet. Stand up and let's say hello.

- Profesor 4: Do you remember how you say [y se toca la cabeza].

- Profesor 4: Repeat after me "Rule number 1: Listen to the teacher", "Rule number 2: Raise your hand" [acompaña la expresión con lenguaje corporal].

Respecto de los estudiantes, se observó una predisposición positiva de ellos a utilizar la lengua extranjera, especialmente en el contexto de saludo y despedida. En muchas ocasiones, saludaban incluso a los investigadores con expresiones como "Miss Andrea! How are you today?". Asimismo, se observó que comprendían numerosas instrucciones básicas en inglés, como "Open your books at page XX", "Let's sing a song!", "Please, be quiet", "Stand up", "Sit down", etc. También eran capaces de producir expresiones orales espontáneas como "See you next month, Miss Andrea", "I don't understand" o "Miss, how do you say acelga in English?" 
Finalmente, en relación con la interacción entre el profesor, el programa y los estudiantes, se encontró que las habilidades más desarrolladas en la sala de clases son las de comprensión, es decir, las habilidades auditiva y lectora, lo que concuerda con la literatura (Lizasoain \& Ortiz de Zárate, 2009). El tiempo de clase se utiliza para escuchar los videos o al profesor, y leer y escribir en el libro de texto y guías. Sí se fomenta la producción, pero esta no es creativa la mayor parte del tiempo, sino que altamente controlada. La habilidad oral se reduce a la repetición y lectura en voz alta, mientras que la escrita, a la completación de oraciones y reproducción de los textos de la pizarra en el cuaderno.

\subsection{Cuestionarios}

Cinco de los siete profesores de las escuelas participantes contestaron el cuestionario, por lo que este solo se analizó superficialmente y en términos cualitativos. Se considera importante incluir estos resultados aquí, puesto que hicieron eco en el Programa Inglés Abre Puertas (PIAP) y su administración del recurso It's my Turn. En relación con al impacto del programa en el aprendizaje del inglés, todos los docentes indicaron una influencia positiva, especialmente en el aprendizaje de vocabulario. Esto es, se reconoce que los estudiantes aprenden y pueden usar el contenido léxico presentado por el programa en actividades controladas. Al respecto, uno de los profesores señaló que "la verdad es que en el caso de quinto básico es fácil de trabajar el libro". Sin embargo, otros dos profesores se refirieron a debilidades del programa como que "falta mas orden en los contenidos que se enseñanan [sic]" o que "no apunta a los intereses de los estudiantes de hoy".

Los docentes también ordenaron de mayor a menor las habilidades que propicia It's my Turn, manifestando que la más fomentada es la habilidad auditiva, seguida por las habilidades de producción oral y escrita y, finalmente, la lectora. En consecuencia, existe coherencia entre estos resultados y los provenientes de las pruebas y observaciones, mediante las cuales se encontró que los estudiantes escriben como escuchan y son más bien pasivos respecto del contenido. 
El impacto adjudicado a ImT en el aprendizaje del inglés tiene directa relación con la evaluación que hacen los docentes del recurso mismo y del papel del PIAP. Al igual que con lo observado en el aula, los profesores expresaron que utilizan el recurso en distintos grados, variando desde $100 \%$ a $25 \%$. Esto porque, según una profesora que lo utiliza el 100\% del tiempo, "es una programa que usa estrategia factible de desarrollar en la sala de clases donde no hay recursos [sic]". Asimismo, los profesores que lo usan menos del 100\% advierten que "es necesario reforzar con otras actividades" o que "los videos traen demasiados temas, entonces se deben realizar otras actividades que repacen los temas por orden [sic]".

Finalmente, los docentes manifiestan que las capacitaciones ofrecidas por el PIAP para el uso del recurso son útiles, pero falta información. A este respecto, aunque no se indica qué tipo de información, en las visitas a las escuelas en el marco de esta investigación, en ocasiones los profesores expresaron desconocer la existencia de algunos elementos del programa, como el cuaderno de planificaciones o el de evaluaciones. Asimismo, los docentes sugirieron no utilizar ImT en escuelas donde los docentes son especialistas en el área -esto es, con título de profesor de inglés-y "enviar[...] los textos oficiales del ministerio por una mejor articulacion de contenidos [sic]", refiriéndose al recurso que se utiliza en los establecimientos urbanos. Esta información fue comunicada al PIAP al término de este estudio.

\section{Discusión: efectividad de It's my Turn}

Debido a que no se puede determinar si es el recurso, su utilización y/o el mayor o menor uso del español o el inglés lo que incide en el aprendizaje con base en una sola variable (las notas de entrada y salida), a continuación se puede observar la combinación de las variables para cada escuela (Tabla 3). 
118 EVALUACIÓN DE IT'S MY TURN, UNA HERRAMIENTA DE AUTOAPRENDIZAJE DE INGLÉS COMO LENGUA EXTRANJERA PARA CONTEXTOS RURALES DE CHILE - A. Lizasoain, A. Ortiz de Zárate, M. Véliz, A. Luci y J. Rojas-Mora

Tabla 3: Significancia estadística, utilización de It's my Turn y uso de L1 y LE

\begin{tabular}{|c|c|c|c|c|}
\hline Escuela & $\begin{array}{l}\text { Significancia } \\
\text { estadística }\end{array}$ & MS-ME & $\begin{array}{l}\text { Utilización de It's my } \\
\text { Turn }\end{array}$ & Uso de L1 y LE \\
\hline \multirow{2}{*}{ Los Huertos } & \multirow{2}{*}{$<0,0001$} & \multirow{2}{*}{1,19} & \multirow{2}{*}{$\begin{array}{l}\text { Uso sistemático y } \\
\text { exclusivo }\end{array}$} & $\begin{array}{l}\text { Profesora con nivel } \\
\text { intermedio alto (B2) }\end{array}$ \\
\hline & & & & $\begin{array}{l}\text { Uso de L1 y LE en igual } \\
\text { proporción }\end{array}$ \\
\hline \multirow[t]{2}{*}{ Chobo A } & \multirow{2}{*}{$<0,0001$} & \multirow[t]{2}{*}{1,14} & \multirow{2}{*}{$\begin{array}{l}\text { Uso parcial y otras } \\
\text { estrategias, sobre todo } \\
\text { corporales }\end{array}$} & $\begin{array}{l}\text { Profesora con nivel } \\
\text { intermedio (B1) }\end{array}$ \\
\hline & & & & Uso de LE mayormente \\
\hline \multirow{2}{*}{ San Andrés } & \multirow{2}{*}{0,0003} & \multirow{2}{*}{0,93} & \multirow{2}{*}{$\begin{array}{l}\text { Uso parcial y otras } \\
\text { estrategias }\end{array}$} & $\begin{array}{l}\text { Profesora con nivel } \\
\text { intermedio (B1) }\end{array}$ \\
\hline & & & & $\begin{array}{l}\text { Uso de L1 y LE en igual } \\
\text { proporción }\end{array}$ \\
\hline \multirow[b]{2}{*}{ El Romero } & \multirow[b]{2}{*}{0,02} & \multirow[b]{2}{*}{0,76} & \multirow[b]{2}{*}{ Sin uso } & $\begin{array}{l}\text { Profesora con nivel } \\
\text { intermedio alto (B2) }\end{array}$ \\
\hline & & & & $\begin{array}{l}\text { Uso de L1 y LE en igual } \\
\text { proporción, con traducción } \\
\text { inmediata a L1 }\end{array}$ \\
\hline \multirow[b]{2}{*}{ El Ciruelo } & \multirow[b]{2}{*}{0,02} & \multirow[b]{2}{*}{0,58} & \multirow[b]{2}{*}{ Sin uso } & $\begin{array}{l}\text { Profesora con nivel } \\
\text { intermedio alto (B2) }\end{array}$ \\
\hline & & & & $\begin{array}{l}\text { Uso de L1 y LE en igual } \\
\text { proporción, con traducción } \\
\text { inmediata a L1 }\end{array}$ \\
\hline \multirow[b]{2}{*}{ Jesucristo } & \multirow[b]{2}{*}{0,08} & & \multirow{2}{*}{$\begin{array}{l}\text { Uso sistemático y } \\
\text { exclusivo }\end{array}$} & $\begin{array}{l}\text { Profesora con nivel } \\
\text { intermedio alto (B2) }\end{array}$ \\
\hline & & & & $\begin{array}{l}\text { Uso de L1 y LE en igual } \\
\text { proporción, con traducción } \\
\text { inmediata a L1 }\end{array}$ \\
\hline \multirow{2}{*}{ Santa Ana } & \multirow{2}{*}{0,11} & & \multirow{2}{*}{$\begin{array}{l}\text { Uso parcial (solo libro de } \\
\text { texto) y otras estrategias }\end{array}$} & $\begin{array}{l}\text { Profesor con nivel } \\
\text { intermedio (B1) }\end{array}$ \\
\hline & & & & $\begin{array}{l}\text { Mayor uso de L1 que } \\
\text { de LE }\end{array}$ \\
\hline Chobo B & 0,23 & & $\begin{array}{l}\text { Uso parcial y otras } \\
\text { estrategias }\end{array}$ & $\begin{array}{l}\text { Mayor uso de L1 que } \\
\text { de LE }\end{array}$ \\
\hline \multirow[b]{2}{*}{ El Melón } & \multirow[b]{2}{*}{0,66} & & \multirow[b]{2}{*}{ Sin uso } & $\begin{array}{l}\text { Profesora con nivel } \\
\text { intermedio alto (B2) }\end{array}$ \\
\hline & & & & $\begin{array}{l}\text { Uso de L1 y LE en igual } \\
\text { proporción, con traducción } \\
\text { inmediata a L1 }\end{array}$ \\
\hline \multirow{2}{*}{ Los Milagros } & \multirow{2}{*}{0,85} & & \multirow{2}{*}{$\begin{array}{l}\text { Uso sistemático y otras } \\
\text { estrategias }\end{array}$} & $\begin{array}{l}\text { Profesora con nivel } \\
\text { avanzado (C1) }\end{array}$ \\
\hline & & & & $\begin{array}{l}\text { Mayor uso de L1 que } \\
\text { de LE }\end{array}$ \\
\hline
\end{tabular}


Según los datos cualitativos organizados en la Tabla 3, las distintas combinaciones de las variables efectivamente inciden en los resultados cuantitativos. Al respecto, parece ser que un mayor uso del español que del inglés, independientemente de la medida de utilización de $\operatorname{ImT}$, tiene un impacto negativo en el aprendizaje de los estudiantes. Por ejemplo, la escuela Los Huertos obtuvo los mejores resultados, con la combinación uso sistemático de ImT/uso proporcional de Ll y LE (sin traducción inmediata de por medio). Por el contrario, la escuela que obtuvo una menor diferencia entre las pruebas de entrada y salida, Los Milagros, presenta la combinación uso sistemático de ImT/mayor uso de Ll. También se observa que en ninguna de las cinco escuelas con mejores resultados se presenta la variable mayor uso de L1, mientras que este fenómeno sí se observa en tres de las cinco escuelas con los resultados más bajos. Consecuentemente, se puede afirmar que un mayor uso del español en la clase perjudica el aprendizaje de la lengua extranjera.

Lo anterior, sin embargo, no explica por qué las escuelas Jesucristo y El Melón obtuvieron resultados poco significativos si las profesoras no utilizaron más la Ll que la LE. Por el contrario, ambas tienen en común la variable uso de Ll y LE en igual proporción, aunque con traducción inmediata a L1, pero se distinguen por el uso sistemático y el "no uso" del recurso. Con base en estas dos escuelas, se podría decir que es ImT y no la lengua utilizada lo que influye positivamente en el aprendizaje del inglés, dado que la escuela Jesucristo obtuvo mejores resultados que la escuela El Melón y utilizó el recurso de forma sistemática. No obstante, como ya se vio, hubo escuelas que lo usaron sistemáticamente y obtuvieron resultados tanto significativos como no significativos. En consecuencia, cabe preguntarse “¿Es ImT efectivo para el autoaprendizaje de inglés en el contexto rural de la Región Metropolitana?"

A partir del análisis anterior, se puede afirmar que It's my Turn es efectivo para el aprendizaje del inglés, independientemente de si su uso es sistemático o no, dado que todos los estudiantes que estuvieron expuestos al recurso aprendieron inglés en mayor o menor medida. Es decir, fueron capaces de comprender y reconocer los elementos lexicogramaticales cubiertos en clases. En este sentido, se puede 
considerar como un recurso de mediación del aprendizaje (Chiappe, 2009), tal y como lo son un libro de texto, una pizarra o un cuaderno. Sin embargo, no se evidenció adquisición, puesto que en general los estudiantes no pudieron crear algo nuevo con lo aprendido (Krashen, 1981, 1985; Lantolf, 2013).

Se reveló, además, que el profesor es fundamental en el proceso de aprendizaje (Lantolf, 2013). Es decir, ningún recurso TIC puede reemplazarlo, como insinúan García et al. (2012). En otras palabras, pese a lo que plantea el Mineduc (2016a), con respecto de ofrecer a los profesores la oportunidad de aprender inglés junto con sus estudiantes, si los docentes no dominan la lengua y/o no la usan en el aula, los estudiantes aprenden menos que aquellos que sí están expuestos a diferentes modos de la lengua extranjera, tal y como también se demostró en la prueba estandarizada aplicada a nivel nacional en 2004. Como afirma Lantolf (2013), el profesor es el responsable del aprendizaje, quien guía al alumno mediante una orquesta de actividades. En consecuencia, si el recurso está pensado especialmente para profesores que no son del área o no hablan inglés con proficiencia-intermedia, a lo menos-, ImT no resulta fundamental para el contexto rural de la Región Metropolitana. Por otro lado, en los cuestionarios se señaló que algunos establecimientos considerados rurales no deberían ser tratados como tales. Cabe destacar aquí que, con base en la información recabada en este estudio, el PIAP decidió ofrecer a todas las escuelas rurales del país la posibilidad de elegir entre el programa It's my Turn y el material didáctico pensado para los profesores especialistas a partir de 2015.

Los resultados también apuntan a que, para lograr el aprendizaje del inglés, el uso predominante del idioma en el aula es fundamental. De otra forma, el estudiante queda privado de contenido lingüístico auténtico (Krashen, 1985; Krashen \& Terrel, 1977), sobre todo en contextos rurales, en los que no suele haber acceso a TV cable ni Internet y, por lo tanto, no se está en contacto permanente con fuentes en lengua inglesa.

Es necesario advertir que no se trata de prohibir la lengua materna en el aula de ILE, puesto que se comprende que su uso es 
necesario para tratar temas conductuales, afectivos, administrativos, etc., así como también para tratar tópicos relativos a la enseñanza y aprendizaje. En relación con el uso de la lengua materna, se observó además que la traducción inmediata del inglés al español resulta perjudicial para el aprendizaje si la práctica es sistemática. Algunos profesores observados en este estudio acostumbraban instruir, explicar o preguntar en inglés y traducir seguidamente, sin esperar la reacción de los estudiantes. En este caso, estos últimos no debían realizar ningún esfuerzo cognitivo, lo cual resulta fundamental para el aprendizaje/adquisición de una lengua (Lantolf, 2013).

En esta línea, es iluminador referirse al caso de la escuela Chobo A de María Pinto, que presenta un comportamiento diferente al de las demás. En primer lugar, tiene el mayor número de estudiantes de las diez escuelas $(N=28)$, lo que podría interpretarse como un obstáculo. En segundo lugar, su profesora demostró tener un nivel de inglés intermedio (B1), en comparación con otras profesoras que demostraron un nivel intermedio alto (B2). En tercer lugar, la profesora hizo un esfuerzo permanente por comunicarse en inglés la mayor parte del tiempo con los estudiantes, utilizando el español como último recurso. Finalmente, la profesora fue la única que combinó estrategias didácticas tradicionales (libro de texto, trabajo en el cuaderno desde el escritorio) con estrategias más innovadoras, como técnicas dramáticas y TIC. En sus clases, los estudiantes tenían una participación activa en situaciones de comunicación real. "Utilizo otro approach en el que busco la práctica oral por sobre todo, y por otra parte no poseo los cd interactivos por lo cual selecciono las actividades que me sirven más [sic]", menciona esta profesora. Todos estos elementos condujeron a la escuela a obtener el cuarto lugar en rendimiento en la prueba de salida, contra toda expectativa. De lo anterior se desprenden otras variables que podrían influir en la efectividad de ImT para el aprendizaje, como son la calidad de las interacciones -en general baja en Chile (Sun et al., 2011) - y el manejo de grupo. Mediante It's my Turn y el uso predominante del inglés, la profesora buscó que los estudiantes utilizaran su conocimiento nuevo inmediatamente y de forma activa. En este sentido, el recurso TIC se convirtió en un mediador del aprendizaje (Chiappe, 2009). 
Resumiendo hasta aquí, podemos respaldar que $\operatorname{ImT}$ es un programa efectivo para aprender inglés, pero no bajo la premisa del autoaprendizaje, ya que los estudiantes no aprenden solos. Es decir, se trata de un recurso didáctico como cualquier otro, que facilita la labor del profesor. En virtud de esto, también podemos agregar que esta herramienta TIC, en particular, promueve el aprendizaje de lenguas extranjeras, en concordancia con García et al. (2012), Clavijo, Quintana y Quintero (2012), Warschauer et al. (2004), quienes defienden el uso de tecnologías de la información y la comunicación en contextos educacionales. Pese a lo expresado por un profesor participante, los estudiantes aprenden con naturalidad en un entorno que les resulta familiar y acorde a sus intereses (Fernández, 2011; White \& Cornu, 2010; Jukes \& Dosaj, 2006), conformado por la sala de clases, el pizarrón, un computador, un proyector, música, libros de estudio, diccionarios, etc. Esto significa que para abarcar distintos intereses y estilos de aprendizaje, es deseable -sino ineludible (Chiappe, 2009; Castells, 1997)- incluir las TIC en la sala de clases; de esta forma, no solo capturamos la atención de los estudiantes, sino que también formamos ciudadanos alfabetos en términos digitales (Warschauer et al., 2004).

\section{Conclusiones}

En el presente estudio se buscó evaluar la efectividad de un programa de autoaprendizaje de inglés como lengua extranjera, It's my Turn, lanzado en 2010 por el Ministerio de Educación de Chile para escuelas rurales de todas las regiones. Se descubrió que el programa puede ser eficaz para el aprendizaje del inglés, dadas ciertas condiciones. Entre estas se encontraron una utilización medianamente sistemática de ImT y un mayor uso de la lengua extranjera versus la lengua materna en el aula.

En relación con el uso sistemático del recurso, se percibió que este también es efectivo si se complementa con las estrategias propias de los docentes, especialmente cuando estas involucran la participación activa del estudiante, ya sea a través de su desplazamiento por la sala (otros puestos o la pizarra) o mediante trabajos que requieren el uso del cuerpo. En este sentido, ImT se presenta como un recurso de mediación del aprendizaje como cualquier otro más tradicional. 
Con respecto al mayor uso de la LE en el aula, se observó que el uso de la Ll no es perjudicial para el aprendizaje, siempre y cuando no se utilice en mayor grado que la LE y no sea parte de la práctica de la traducción inmediata del inglés al español. En resumen, el papel del profesor, principal fuente de contenido lingüístico y guía del aprendizaje, no puede obviarse.

Además, se notó cierto grado de satisfacción tanto con el programa como con el papel del Mineduc, aunque los datos apuntan a la necesidad de realizar algunas mejoras. Por ejemplo, los docentes indicaron que una sola lección contiene demasiados contenidos sin una relación ni progresión claras que faciliten la enseñanza y el aprendizaje de estos. Al respecto, se sugiere explicar esta lógica en las capacitaciones que se ofrecen bianualmente a los profesores que utilizan el programa. Al mismo tiempo, se encontró que estos no cuentan con parte de la información atingente a las mejoras del programa, como la reciente integración del cuaderno de planificación y el cuadernillo de evaluaciones. Asimismo, algunas escuelas no disponían de los dispositivos necesarios para ver los videos o escuchar el audio, como computadores, proyectores, parlantes, etc. Una profesora incluso tuvo que comprar su propio proyector.

En relación con el uso de TIC en la sala de clases, se observa que estas efectivamente tienen el potencial para fomentar el aprendizaje, pero que al parecer no resultan fundamentales para el sector inglés. Sin embargo, esto puede deberse a la forma cómo se usan y no a su mera naturaleza. Al respecto, se encontró que en ocasiones la instalación de computadores y proyectores retrasaba el comienzo de la clase, lo que conducía a una pérdida de instancias de aprendizaje, así como a problemas de disciplina. Al mismo tiempo, contrario a lo que se señala en la literatura, no se observó que It's my Turn fomentara una interacción significativa entre los estudiantes y el recurso, así como tampoco el trabajo colaborativo, ni al autoaprendizaje. Por el contrario, las clases siguen teniendo la estructura clásica de pupitres en filas organizadas por un profesor que lidera unos intercambios que en general no conducen a aprendizajes reales, sino que solo sirven para verificar que los estudiantes comprendieron una instrucción o pueden repetir una expresión lingüística, lo que se condice con los 
resultados de la Evaluación Docente (Sun et al., 2011) así como con las pruebas de 2004 .

Lo anterior puede explicarse con base en la reciente incorporación formal de las TIC en las salas de clase chilenas. Esta comenzó a mediados de la década de los noventa, a través del proyecto Enlaces del Mineduc (Hinostroza, Hepp \& Cox, 2009), cuyo fin era generar una red educacional en el nivel nacional entre todos los establecimientos escolares subvencionados de Chile, y luego integrar las TIC al currículo (Mineduc, 2016d). Esto último a través del desarrollo de competencias pedagógicas para utilizar las TIC en el aula y la diseminación de modelos de uso de TIC para la enseñanzaaprendizaje, entre otras medidas (Hinostroza \& Brun, 2010). Desde entonces, los establecimientos escolares comenzaron un proceso, no poco accidentado, de transformación a través de la incorporación y desarrollo de TIC que, como muestra el estudio, aún no ha culminado.

En esta línea, consciente del rol transformador del profesor, en 2006, Enlaces implementó los denominados Estándares TIC para la Formación Inicial Docente (Mineduc, 2011), los cuales abordan de forma particular aspectos vinculados con el desarrollo profesional en TIC. No obstante, desafortunadamente, estos estándares no tienen carácter obligatorio, sino que son sugeridos, por lo que el éxito relativo de la incorporación de las TIC en educación ha descansado en la decisión, un tanto aislada, del agente que más influye en el proceso de enseñanza-aprendizaje: el profesor. Es por ello que la política pública de las TIC pasa también por analizar lo que ha ocurrido en la incorporación de las TIC en la formación de profesores. Lo anterior va acompañado de otros desafíos, como resolver la falta de tiempo para la implementación y familiarización con las TIC por parte de los agentes de cambio, la escasez de modelos de apoyo para dicha implementación, la casi nula infraestructura para incluirlas y el peso de la tradición (Bakir, 2015; Hinostroza \& Brun, 2010; Lizasoain \& Ortiz de Zárate, 2009; Brzycki \& Dust, 2005).

Ante esta realidad, se hace un llamado a evaluar la implementación y eficacia de otros programas de enseñanza en Chile -no solo mediados por TIC-, con el fin de mejorar la calidad 
de la educación en contextos rurales y urbanos con base en evidencia empírica, así como a generar métodos de enseñanza-aprendizaje que digan relación con las diferentes realidades socioculturales del país.

\section{Referencias}

Álvarez, G. y Álvarez, G. (2012). Hacia una propuesta de análisis semiótico integral de ambientes virtuales de aprendizaje. Onomázein, 25(1), 219-239.

Arancibia, M. (2002). Transformaciones en las organizaciones educativas que posibiliten aprendizajes transdisciplinarios con utilización de recursos informáticos. Estudios Pedagógicos, 28, 143-156. http:// dx.doi.org/10.4067/s0718-07052002000100008

Bakir, N. (2015). An exploration of contemporary realities of technology and teacher education: Lessons learned. Journal of Digital Learning in Teacher Education, 31(3), 117-130. http://dx.doi.org/10.1080/2153 2974.2015.1040930

Becchi, C. (2012). It's my Turn: Evaluation of the effectiveness of a rural selflearning English program (Tesis de pregrado inédita). Universidad Austral de Chile, Valdivia, Chile.

Brown, D. (2004). Language assessment: Principles and classroom practices. New York: Pearson.

Brzycki, D. \& Dust, K. (2005). Overcoming barriers to technology use in teacher preparation programs. Journal of Digital Learning in Teacher Education, 13(4), 619-641.

Cárcamo, L., Cladellas, R. y Castelló, A. (2011). Motivación y estimación del tiempo en el uso de herramientas de internet informacionales y dialógicas. El Profesional de la Información Journal, 20(1), 25-31. http://dx.doi.org/10.3145/epi.2011.ene.03

Cárcamo, L., Cladellas, R. y Estaún, S. (2007). Estimación de tiempo en estudiantes secundarios chilenos frente a una tarea espacial desarrollada en formatos de papel y ordenador. Estudios Pedagógicos, 33(2), 27-44. http://dx.doi.org/10.4067/s0718-07052007000200002

Castells, M. (1997). La era de la información: economía, sociedad y cultura. La sociedad red. Madrid: Alianza.

Chiappe, A. (2009). Acerca de lo pedagógico en los objetos de aprendizaje. Reflexiones conceptuales hacia la construcción de su estructura teórica. Estudios Pedagógicos, 35(1), 261-272. http://dx.doi. org/10.4067/s0718-07052009000100016 
Clavijo, A., Quintana, A. y Quintero, L. (2012). Enseñanza del inglés y medios digitales: nuevos retos y posibilidades para la escuela. Bogotá: Editorial UD.

Cohen, L., Manion, L., \& Morrison, K. (2007). Research methods in education. London and New York: Routledge.

Creswell, J. (2009). Research design. Qualitative, quantitative, and mixed methods. Los Angeles, London and New York: Sage.

Fernández, C. (2011). Elementos que contribuyen a la construcción de un modelo curricular para la integración de TIC en el segundo ciclo de educación parvularia. REXE, 10(20), 109-125.

Flores, P. (2012). Aprendizaje mediado por tecnologías: impacto de estrategias pedagógicas apoyadas por la web en estudiantes de la escuela México Valdivia, Región de los Ríos (Tesis de magíster inédita). Universidad Austral de Chile, Valdivia, Chile.

Flyman-Mattsson, A. (1999). Students' communicative behavior in a foreign language classroom. Working Papers, 47, 39-57.

García, J., Ferrerira, A. y Morales, S. (2012). Autonomía en el aprendizaje de lenguas extranjeras en contextos de enseñanza mediados por la tecnología. Onomázein, 25(1), 15-50.

Gass, S. \& Mackey, A. (2012). Introduction. En S. Gass \& A. Mackey (Eds.), The Routledge handbook of second language acquisition (pp. 1-4). New York: Routledge.

Hatch, E. \& Farhady, H. (1982). Research design and statistics for applied linguistics. Rowley, London and Tokio: Newbury House.

Hepp, P. (10-11 de enero de 2013). Comunicación presentada en el Seminario de profesores innovadores. Ideas festival. Valdivia, Región de los Ríos, Chile: Universidad Austral de Chile.

Hinostroza, J. \& Brun, M. (2010). International association for the evaluation of educational achievement. Recuperado de: http://www.iea.nl/fileadmin/ user_upload/IRC/IRC_2010/Papers/IRC2010_Hinostroza_Brun.pdf

Hinostroza, J., Hepp, P., \& Cox, C. (2009). Policies and practices on ICT in education in Chile: Enlaces. En T. Plomp, R. Anderson, N. Law, \& A. Quale (Eds.), Cross-national information and communication technology: Policies and practices in education (pp. 153-170). Greenwich: Information Age Publishing.

Jukes, I. \& Dosaj, A. (2004). Understanding digital children (DKs). Teaching \& learning in the new digital landscape. Recuperado de http://www.wright. edu/ marguerite.veres/786syl/growingupdigit.pdf 
Krashen, S. (1981). Second language acquisition and second language learning. Oxford and New York: Pergamon Press Inc.

Krashen, S. (1985). The input hypothesis: Issues and implications. London and New York: Longman.

Krashen, S. \& Terrel, T. (1977). The natural approach (language acquisition in the classroom). Hayward, CA: Alemany Press.

Lantolf, J. (2013). Sociocultural theory and the dialectics of L2 learner autonomy/agency. En P. Benson \& L. Cooker (Eds.), The applied linguistic individual: Sociocultural approaches to identity, agency and autonomy (pp. 17-31). Sheffield and Bristol: Equinox.

Lizasoain, A. \& Becchi, C. (2014). Evaluation of a rural self-learning English program in Chile. Enjoy Teaching Journal, 2(2), 4-18.

Lizasoain, A. \& Ortiz de Zárate, A. (2009). Efficiency and effectiveness of drama techniques. Recuperado de http://eric.ed.gov/?id=ED523207

Lizasoain, A., Ortiz de Zárate, A., Walper, K. y Yilorm, Y. (2011). Estudio descriptivo y exploratorio de un taller de introducción a las técnicas teatrales para la enseñanza/aprendizaje de una lengua extranjera. Estudios Pedagógicos, 37(2), 123-133. http://dx.doi.org/10.4067/ s0718-07052012000200010

Ministerio de Educación de Chile, Mineduc. (2010). It’s my Turn. Guía didáctica. $5^{\text {to }}$ básico. Santiago de Chile: Longman.

Ministerio de Educación de Chile, Mineduc. (2011). Competencias TIC para docentes. Recuperado de http://www.enlaces.cl/portales/ tp3197633a5s46/documentos/200707191420080.Estandares.pdf

Ministerio de Educación de Chile, Mineduc. (2014). Base de datos. Recuperado de http://data.mineduc.cl

Ministerio de Educación de Chile, Mineduc. (2014). Indicadores educativos para la comunidad. Recuperado de http://data.mineduc.cl

Ministerio de Educación de Chile, Mineduc. (2016a). Programa Inglés Abre Puertas. Recuperado de http://ingles.mineduc.cl

Ministerio de Educación de Chile, Mineduc. (2016b). ¿Qué es It's my Turn? Recuperado de: http://www.ingles.mineduc.cl/index2.php?id_ portal=49\&id_seccion=3370\&id_contenido=13776

Ministerio de Educación de Chile, Mineduc. (2016c). Estándares orientadores para carreras de Pedagogía en Inglés. Recuperado de http://www. cpeip.cl/index2.php?id_seccion=4888\&id_portal=41\&id_ contenido $=27356$ 
128 EVALUACIÓN DE IT'S MY TURN, UNA HERRAMIENTA DE AUTOAPRENDIZAJE DE INGLÉS COMO LENGUA EXTRANJERA PARA CONTEXTOS RURALES DE CHILE - A. Lizasoain, A. Ortiz de Zárate, M. Véliz, A. Luci y J. Rojas-Mora

Ministerio de Educación de Chile, Mineduc. (2016d). Enlaces. Recuperado de http://www.enlaces.cl/index.php?t=44\&i $=2 \& \mathrm{cc}=174 \& \mathrm{tm}=2$

Nunan, D. (1992). Research methods in language learning. New York, Oakleigh: CUP.

Páez, A. (2012). PowerPoint Chilean curriculum. Santiago de Chile: Universidad Diego Portales.

Pérez, J. M. (2000). Comunicación y educación en la sociedad de la información. Barcelona: Paidós.

Prensky, M. (2001). Digital natives, digital immigrants Part II: Do they really think differently? Horizon, 9(6), 1-6. http://dx. doi. org/10.1108/10748120110424843

Pumarino, J. I. (2016). Generador de escalas de notas. Recuperado de http:// escaladenotas.cl/

Red Maestros de Maestros (2004). Recuperado de: http://www.rmm.cl/ usuarios/jriva/doc/200609251337550cambridge_resultados_simce. pdf

Richards, J. \& Rogers, T. (2008). Approaches and methods in language teaching. New York: Cambridge University Press.

Stenhouse, L. (1983). Authority, education and emancipation. London: Heinemann.

Stryker, S. \& Leaver, B. (1997). Content-based instruction in foreign language education: Models and methods. Washington, D.C.: Georgetown University Press.

Sun, Y., Calderón, P., Valerio, N., y Torres, P. (2011). La implementación de la evaluación docente. En J. Manzi, R. González, e Y. Sun (Eds.), La evaluación docente en Chile (pp. 63-91). Santiago de Chile: Mide UC.

Warschauer, M., Shetzer, H., \& Meloni, C. (2004). Internet for english teaching. Washington D.C.: TESOL.

White, D. y Le Cornu, A. (2011). Visitantes y residentes: una nueva tipología para el usuario digital. Recuperado de https://docs.google.com/ file/d/0B9vhK_eXYvneZ2VBeFd3WjN2OGM/edit

Recibido: 16/04/2015

Aceptado: 11/03/2016 